\title{
Bronchiectasis in Indigenous children in remote Australian communities
}

\author{
Anne B Chang, Keith Grimwood, E Kim Mulholland, Paul J Torzillo, for the Working Group
} on Indigenous Paediatric Respiratory Health

BRONCHIECTASIS is a progressive disease process characterised by dilated, thick-walled bronchi caused by chronic bacterial or viral infection and inflammation. It is now rarely encountered in developed countries, except in patients with cystic fibrosis (CF), or in association with other underlying causes such as ciliary dyskinesia, immune deficiency or focal lung abnormalities. In contrast, bronchiectasis remains common among Indigenous populations, involving $1 \%-2 \%$ of all children, with symptoms frequently dating back to infancy. ${ }^{1,2}$

The burden of ill-health from respiratory disease among Indigenous Australians is significant. ${ }^{3}$ Respiratory disease is the commonest preventable, defined cause of infant deaths, ${ }^{4}$ the most common disorder in children aged under 5 years in Northern Territory hospital morbidity data, ${ }^{5}$ and the second-highest cause of death among adults. The true prevalence of bronchiectasis and associated respiratory illness in Indigenous children in Australia is unknown, but is likely to be disproportionately high in rural and remote communities. A recently completed central Australian study estimated the rate of bronchiectasis (confirmed by highresolution computed tomography [HRCT] ${ }^{6,7}$ ) in children aged under 15 years as 147 per 10000 Aboriginal children (unpublished data), a rate 40-fold greater than that for $\mathrm{CF}$ in non-Indigenous populations (35 per 100000$).{ }^{8}$ However, there are no programs or resources to manage these patients, and even a perception that "nothing can be done" - similar to the situation that existed for patients with $\mathrm{CF}$ several decades ago.

Consequently, the Working Group on Indigenous Paediatric Respiratory Health developed consensus recommendations. The consensus process is summarised in Box 1, our

\footnotetext{
Northern Territory Clinical School, Flinders University, Alice Springs, NT.

Anne B Chang, MPHTM, PhD, FRACP, Associate Professor of Paediatrics and Respiratory Physician.

Wellington School of Medicine and Health Sciences, Wellington, New Zealand.

Keith Grimwood, MD, FRACP, Professor of Paediatrics and Child Health and Paediatric Infectious Diseases Physician.

Centre for International Child Health, Royal Children's Hospital, Parkville, VIC.

E Kim Mulholland, MD, FRACP, Head, and Professorial Fellow. University of Sydney and Royal Prince Alfred Hospital, 420/RPAH Medical Centre, Newtown, NSW.

Paul J Torzillo, MB BS, FRACP, Clinical Associate Professor; and Medical Director, Nganampa Health Council.

Reprints will not be available from the authors. Correspondence: Associate Professor Anne B Chang, Department of Respiratory Medicine, Royal Children's Hospital, Herston, QLD 4029. abchang @mac.com
}

\begin{abstract}
- The rates of bronchiectasis for Indigenous children from remote Australian communities are unacceptably high, with one study showing 14.7/1000 Aboriginal children.

- Children with bronchiectasis need to be identified early for optimisation of medical treatment. Under-reporting of cough is common. Bronchiectasis should be suspected in children with recurrent bronchitis or pneumonia, and when, despite appropriate therapy, pulmonary infiltrates or atelectasis persist 12 weeks beyond the index illness.

- During acute infective episodes, oral antibiotics and chest physiotherapy to clear the airways should produce prompt resolution; otherwise, hospitalisation is necessary.

- Management follows the cystic fibrosis model of regular review, encouragement of physical activity, optimising nutrition, maintenance of immunisation and avoidance of environmental toxicants, including passive smoke exposure.

- Successful management and prevention of bronchiectasis will require improvements in housing, nutrition, and education, as well as access to comprehensive healthcare services, with coordination between primary and hospitalbased healthcare providers.
\end{abstract}

MJA 2002; 177: 200-204

recommendations are listed in Box 2 and research goals in Box 3. The recommendations were conditioned by the current incomplete knowledge of the aetiology and disease mechanisms of bronchiectasis, and a lack of large randomised-controlled trials able to detect clinically significant differences for interventions in non-CF bronchiectasis. These limitations were most apparent in children. For these reasons, the consensus recommendations are based on the best available evidence, including extrapolation from studies in CF patients.

\section{Early detection (Box 2, Objective A)}

\section{Diagnosis}

The clinical case definition of bronchiectasis is imprecise and its severity and extent is highly variable, ranging from mild respiratory morbidity to death. Symptoms suggestive of the disease are listed in Box 2. Chest radiographs are relatively insensitive, one study detecting fewer than $50 \%$ of patients with bronchography-proven bronchiectasis. ${ }^{6}$ Peribronchial fibrosis thickens bronchial walls so that they are seen as 


\section{1: Consensus process}

The working group comprised 44 representatives from Australian and New Zealand medical schools, secondary and tertiary paediatric centres (see Acknowledgements). Collectively, the group possessed expertise in Indigenous health, paediatric and respiratory medicine, microbiology, infectious diseases and public health. MEDLINE databases were searched from January 1966 to November 2001 for subject headings (bronchiectasis, bronchitis, suppurative lung, suppurative respiratory disease) and the Cochrane database was also accessed using these headings. Review of references led to identification of relevant articles published before 1966, while experts in the working group identified unpublished data. The first draft of the working group's consensus statement followed a two-day meeting in Alice Springs in August 2001, where the available evidence was presented and reviewed. Suggested revisions were incorporated into subsequent drafts, so that the final draft represents all relevant evidence obtained by the literature search, in conjunction with final consensus

recommendations annotated to reflect the level of consensus among the entire group.

E1: Systematic review of all relevant randomised-controlled trials (RCTs)

E2: Well-designed RCTs

E3: Well-designed cohort or case-control studies

C1: Complete consensus of entire working group

C2: Near-complete consensus (95\% or more of working group)

C3: No consensus

"tramlines", especially in the lower lobes. HRCT optimised with a high-spatial-frequency reconstruction algorithm is the current "gold standard" for identifying bronchiectatic airways. 9 Typical HRCT findings of bronchiectasis include "signet-ring" appearance (dilated bronchus compared with adjacent pulmonary artery), loss of bronchial tapering, and end-expiratory scans showing increased translucency in areas of air trapping from small-airways disease.

\section{Recurrent lower respiratory tract infection}

Infection is an important contributor to later respiratory morbidity, chronic lung disease and pulmonary function abnormalities. ${ }^{10-18}$ The nasopharyngeal spaces of Indigenous central Australian children are heavily colonised by potential respiratory tract bacterial pathogens, ${ }^{19}$ and the attack rates of acute lower respiratory tract infection are similar to those in children in developing countries. ${ }^{20}$ Of 116 children aged under 15 years admitted to Alice Springs Hospital (October 2000 - April 2001) with radiologically defined lobar pneumonia, 58 (50\%) had previously been hospitalised on two or more occasions for acute lower respiratory tract infections. As of June 2001, of 76 children (84 episodes, $64 \%$ of children) reviewed 2-8 months later, 10 (13\%) were diagnosed with bronchiectasis by HRCT in the non-acute state (unpublished data). Given the high rate of respiratory morbidity after hospitalisation in this patient group, clinical follow-up is recommended as a minimum (C1).

\section{Investigations}

The principal aims of investigations in children with suspected bronchiectasis are to confirm the diagnosis, to define

\section{2: Recommendations}

\section{Objective A: To encourage early diagnosis of bronchiectasis}

- Bronchiectasis should be suspected in any child with recurrent lower respiratory tract infection, prolonged moist-sounding cough, exertional dyspnoea, symptoms of reactive airways disease, growth failure, hyperinflation of lungs, chest deformity or digital clubbing (E3). Under-reporting of cough is common and obtaining additional medical information from the local community (clinic staff, notes, carers, health workers) is important (C1).

- For prompt detection of bronchiectasis, all Indigenous children with radiologically proven pneumonia should undergo clinical review $(\mathrm{C} 1)$. If the child is aged $<2$ years, has a history of recurrent bronchitis or pneumonia, atelectasis is present in the initial radiograph, or a chronic cough ( $>4-6$ weeks) is suspected a chest radiograph is performed 6-8 weeks after treatment to ensure complete radiological resolution (C2).

- When, despite treatment, radiological changes persist for more than 3 months, or when symptoms and/or signs suggest the presence of bronchiectasis, chest high-resolution computed tomography scans should be performed in the non-acute state (C1).

\section{Objective B: To optimise medical management to reduce morbidity} and preserve lung function

- Management following CF models of care is recommended (C2). Exacerbations are treated with antibiotics determined by sputum bacteriology (E3). Treatment duration of 2-6 weeks is defined by the child's general health, the amount of sputum and the pathogen cultured (E3). When respiratory secretion collection is not possible, empirical antibiotic therapy is directed at pathogens commonly associated with bronchiectasis (C2).

- Children with bronchiectasis should be reviewed 3-monthly by the most appropriate local doctor, in consultation with other healthcare providers, and have a minimum 6-monthly review by a paediatrician or respiratory physician (C2).

- Immunisation status should be reviewed and updated (C1).

Objective C: To promote public health issues and healthcare delivery

- Exposure to wood smoke and environmental tobacco smoke should be minimised (E3)

- A culture- and expertise-appropriate healthcare delivery model and a coordinated approach among various healthcare providers should be used (C1).

- The socioeconomic determinants of health must be recognised (C1).

the distribution and severity of airway involvement, and to identify familial and treatable causes of bronchiectasis. Chest HRCT should identify congenital lesions and determine the extent and severity of disease. Lung function tests are non-specific, but in older children provide a measure of functional impairment and small-airways involvement. ${ }^{18}$ Other investigations include sputum culture for bacteria and mycobacterial species, sweat testing (although CF is extremely rare in Aboriginal children), Mantoux testing, immune function assessment (differential white-cell count, HIV, immunoglobulins G, A, M, E and IgG subclasses, antibody responses to protein and polysaccharide antigens) and nasal cilia biopsy (C2) (although transient abnormal cilia are often found with nasal space disease, which is a very common occurrence in rural Indigenous children). ${ }^{20}$ For selected patients, bronchoscopy to exclude a foreign body and obstructive bronchiectasis, as well as fluoroscopic 
examination for aspiration, are recommended (C1). The extent of investigations has to be considered on an individual basis for children from remote communities (C1).

\section{Management (Box 2, Objective B)}

Without adequate data to guide management of non-CF bronchiectasis, a similar approach to the management of $\mathrm{CF}^{21,22}$ (and ciliary dyskinesia ${ }^{23}$ ) is recommended (C2). However, it is uncertain whether CF patients, with their multiorgan involvement, exaggerated airway inflammatory responses and accelerated disease progression, serve as the best model of care for those with bronchiectasis from other aetiologies. In addition, the respiratory pathogens involved only partially overlap. ${ }^{21}$

Management of bronchiectasis involves aggressive treatment of recurrent exacerbation, promotion of mucociliary clearance (chest physiotherapy, exercise, etc), a vigilant approach to reduce lung damage from other causes (eg, aspiration), promotion of normal growth, management of psychosocial aspects, and identification and treatment of complications as they arise. ${ }^{21,24}$ Other aspects include regular review, optimising nutrition, maintenance of immunisation and avoidance of environmental toxicants, including passive smoke exposure.

\section{Treatment of infection}

\section{Mechanism of bronchial injury}

It is hypothesised that, in some children, impaired mucociliary clearance after severe or recurrent acute lower respira-

\section{3: Research goals}

\section{Aetiology and disease burden}

- Identify the respiratory disease burden

- Describe aetiological and modifiable risk factors

- Determine whether acute lower respiratory tract infection is adequately treated, with resolution of symptoms and pulmonary infiltrates

- Pursue the relationship between genes, polymorphisms and regulation of the immune response

- Determine the relationship between chronic ear disease and bronchiectasis, and whether nasopharyngeal colonisation by potential respiratory pathogens is a risk factor

- Distinguish whether early and aggressive treatment of prebronchiectatic chronic suppurative lung disease can prevent the development of bronchiectasis

\section{Management}

- Identify the predominant pathogens and determine the best means of collecting respiratory secretions from non-expectorating patients

- Conduct intervention studies to determine the type and duration of antibiotic therapy, stratified by disease severity

- Conduct intervention studies to determine the role of antibiotic suppressive therapy

- Describe the role and best method of chest physiotherapy

- Determine the best outcome measures for young children

- Resolve whether early management preserves lung function tory tract infection allows development of endobronchial infection by microaspiration of nasopharyngeal bacteria, ${ }^{25}$ inflammation and progressive bronchial wall injury. Recurrent inflammation may impair lung growth as well as accelerate respiratory function decline. ${ }^{26,27}$

\section{Infective organisms}

There are no published data on organisms that colonise and cause exacerbations in Indigenous Australian children with bronchiectasis. Haemophilus influenzae, Streptococcus pneumoniae and Moraxella catarrhalis, bacteria that colonise the nasopharynx, cause lower respiratory tract infections and are associated with bronchiectasis in other Indigenous populations. ${ }^{2,19}$ Unlike in CF, ${ }^{28}$ Staphylococcus aureus is uncommon, and opportunistic pathogens like Pseudomonas aeruginosa are usually only found in the sputum of older patients with advanced disease. ${ }^{29,30}$ Sputum cultures may be contaminated by upper-airway organisms and antibiotics should be directed against anticipated pathogens. More invasive testing, for example by induced sputum or bronchial lavage, should be considered if symptoms persist (C2).

\section{Antibiotic therapy}

When bronchiectasis is mild, antibiotics may eradicate the infection, and lung defences keep the airways sterile or bacterial numbers low for prolonged periods. ${ }^{31}$ If airway injury is more severe, the bronchi are chronically infected and symptoms may rapidly return. However, apart from studies on chronic $P$. aeruginosa infection in adults with $\mathrm{CF}$, there are no studies to show that long-term antibiotics prevent disease progression. ${ }^{32}$ It is for this reason, and the risk of selecting resistant organisms, that interval rather than suppressive antibiotic therapy is recommended (C1).

Children with a chronic moist-sounding cough should receive antibiotics such as amoxycillin-clavulanate (or high dose amoxycillin, co-trimoxazole, cefaclor) for 2-6 weeks or until the cough resolves (C1). If the cough does not improve, the patient requires specialist reassessment or hospitalisation, particularly if there is a deterioration in general health, weight loss, reduced lung function or new radiographic infiltrates (C2). Inpatient management includes parenteral antibiotics, such as ampicillin, cefotaxime or ceftriaxone, and intensive physiotherapy (C1). When $P$. aeruginosa is present, ciprofloxacin or combined therapy with an antipseudomonal $\beta$-lactam and an aminoglycoside is preferred (C1). Given the lack of resources in their communities, Indigenous children with moderate or severe bronchiectasis are more likely to require hospitalisation (C2).

Antibiotics in adults with bronchiectasis significantly improve quality-of-life measures, ${ }^{30,33}$ and airway and systemic ${ }^{33}$ inflammatory profiles. ${ }^{33,34}$ Similar information in children is lacking, and there are no data evaluating the effects of long-term antibiotics on acquisition of antibioticresistant pathogens.

\section{Other measures}

Reactive airways disease can be present in children with bronchiectasis, ${ }^{35}$ and the use of asthma medications should 
be individualised $(\mathrm{C} 1)$. There are insufficient data to recommend the regular use of inhaled or oral corticosteroids, ${ }^{36} \beta_{2^{-}}$ agonists, mucolytics or methylxanthines in patients with bronchiectasis.

\section{Chest physiotherapy}

Chest physiotherapy improves airway clearance, but the best method is yet to be defined. Although there are no controlled trials on the role of surgery, it may be considered in those who have passed puberty when bronchiectasis is focal and medical therapy has failed ${ }^{37}(\mathrm{C} 1)$.

\section{Regular review}

Regular review aims to optimise lung growth, minimise respiratory decline, improve quality of life and provide health education (C1). The review should include measurement of pulmonary function (for children aged 6 years or over), assessment and management of pulmonary deterioration and infective exacerbations (sputum and cough changes, exertional dyspnoea), management of complications of bronchiectasis (pulmonary hypertension, chronic hypoxaemia, poor growth, sleep disturbance, reactive airways disease, haemoptysis) and a review of contributory factors (eg, gastro-oesophageal reflux, asthma, environmental smoke exposure). As with successful CF models, ${ }^{21,22}$ a multidisciplinary approach with nursing and allied health expertise (social work, physiotherapy, dietitian) is used. ${ }^{38}$ This requires appropriate health resources and adequate systems that support specific management plans for children and carers (C2). An individualised management protocol is important, with a leader selected from the team to take responsibility for each child (C2).

\section{Vaccination}

Immunisation programs should include vaccination against pneumococcus and influenza, in addition to the routine childhood vaccination schedule (C1). Future vaccine development may allow the introduction of vaccines that protect against non-type $\mathrm{b}$ and non-typable strains of $H$. influenzae, $M$. catarrhalis and respiratory syncytial virus.

\section{Public health issues and healthcare delivery (Box 2, Objective C)}

These extremely important topics cannot be adequately covered in this article. In brief, health is closely linked to socioeconomic factors. ${ }^{39}$ Successful management and prevention of bronchiectasis among Indigenous populations will only be achieved by delivering comprehensive healthcare, accompanied by improvements in housing, nutrition and education, and by alleviation of poverty and unemployment (C1).

The effects of in-utero and ex-utero exposure to environmental tobacco smoke on children's respiratory system include reduced lung function, increased acute lower respiratory tract infection, and middle-ear disease ${ }^{40}$ (E3). Indoor wood smoke increases acute lower respiratory tract infection, demonstrating an exposure-response effect ${ }^{41}$ (E3). Interventions to reduce environmental tobacco smoke and biomass smoke exposure are urgently needed. The associa- tion between maternal education and childhood health indices in developing nations ${ }^{42}$ has not been shown to occur in the Australian Indigenous population. An increased risk of acute lower respiratory tract infection is associated with greater numbers of house occupants, damp housing, macroand micromalnutrition, and inadequate water supply ${ }^{43,44}$ (E3).

No single model of healthcare delivery can be used for all Indigenous communities. A comprehensive and competent primary healthcare service is a prerequisite for the effective delivery of any treatment and disease control program. Education of primary healthcare providers to identify appropriate children for referral and primary management of children with bronchiectasis would be beneficial (C2).

The challenge for health service systems is to find ways to deliver effective, competent and quality healthcare despite problems that include remoteness, endemic poverty, severe educational disadvantage, dysfunctional communities, and extreme comorbidities in both children and their carers.

\section{Conflict of interest}

None identified

\section{Acknowledgements}

The workshop was sponsored by the Alice Springs Hospital Management Board Abbott Australasia, GlaxoSmithKline Australia, Bayer Australia, the Centre for Remote Health, and Flinders University Northern Territory Clinical School.

Participating/contributing members of the Indigenous paediatric healh respiratory workshop

Adelaide: R Antic* (Royal Adelaide Hospital); I J Martin* (Women's \& Children's Hospital.

Alice Springs: A Brown* (Centre for Disease Control); A B Chang*, M Segasothy* J Thurley† (Flinders University Northern Territory Clinical School); S Bollisetty†, T Clothier $^{\star}$, J Erlich*, A L Jaquirey*, C Mathews* ${ }^{\star}$, G Wheaton* (Alice Springs Hospital); D Ewald*, j Wakerman* (Centre for Remote Health); A White* (Remote Health); Richard Limt (general practitioner and member of NT parliament).

Auckland: M I Asher†, C Byrnes* (Starship Children's Hospital).

Brisbane: I B Masters*, P Francis† (Royal Children's Hospital)

Darwin: D Brewster* (Flinders University Northern Territory Clinical School); P Bauert*

G McGuire* (Royal Darwin Hospital); A Leach*, P Morris*, B Currie† (Menzies School of Health Research), V Krause* (Centre for Disease Control).

Melbourne: K Mulholland*, P D Phelan*, P J Robinsont, J Massie† (Royal Children's Hospital)

Newcastle: J Stuart† (John Hunter Hospital).

Perth: L Landaut, P N Le Souëf* (University of Western Australia); P Sly†, S Stick† (Princess Margaret Hospital); D Lehmann* (TVW Telethon Children's Institute, Perth); R Edwards* (Australian Council on Smoking and Health).

Sydney: P Mclntyre*, C Mellist, P Van Asperent (Children's Hospital at Westmead Sydney); J Morton† (Sydney Children's Hospital); P J Torzillo* (Royal Prince Alfred Hospital; and Nganampa Health Council).

Wellington: K Grimwood* (Wellington School of Medicine and Health Sciences)

*Attended meeting and/or presented data and contributed to document. †Contributed to and/or supported document.

\section{References}

1. Maxwell GM, Elliott RB, McCoy WT, Langsford WA. Respiratory infections in Australian Aboriginal children: a clinical and radiological study. Med J Aust 1968; 2: $990-993$

2. Singleton R, Morris A, Redding G, et al. Bronchiectasis in Alaska Native children: causes and clinical courses. Pediatr Pulmonol 2000; 29: 182-187.

3. Australian Bureau of Statistics. Mortality of Aboriginal and Torres Strait Islander Australians. Canberra: ABS, 2000. (Catalogue No. 3315.0.)

4. Dempsey KE, Condon JR. Mortality in the Northern Territory 1979-1997. Darwin Territory Health Services, 1999.

5. d'Espaignet ET, Kennedy K, Paterson BA, et al. From infancy to young adulthood health status in the Northern Territory. Darwin, Territory Health Services, 1998. 
6. Currie DC, Cooke JC, Morgan AD, et al. Interpretation of bronchograms and chest radiographs in patients with chronic sputum production. Thorax 1987; 42: 278-284.

7. Grenier P, Maurice F, Musset D, et al. Bronchiectasis: assessment by thin-section CT. Radiology 1986; 161: 95-99.

8. Massie RJ, Olsen M, Glazner J, et al. Newborn screening for cystic fibrosis in Victoria: 10 years' experience (1989-1998). Med J Aust 2000; 172: 584-587.

9. Webb WR, Muller NL, Naidich DP. Airway diseases. High-resolution CT of the lung. Philadelphia: Lippincott, Williams \& Wilkins, 2001: 467-546.

10. Roberts HR, Wells AU, Milne DG, et al. Airflow obstruction in bronchiectasis: correlation between computed tomography features and pulmonary function tests. Thorax 2000; 55: 198-204.

11. Nikolaizik WH, Warner JO. Aetiology of chronic suppurative lung disease. Arch Dis Child 1994; 70: 141-142.

12. Meeks M, Bush A. Primary ciliary dyskinesia. Pediatr Pulmonol 2000; 29: 307-316.

13. Chang AB, Masel JP, Masters B. Post-infectious bronchiolitis obliterans: clinical, radiological and pulmonary function sequelae. Pediatr Radiol 1998; 28: 23-29.

14. Becroft DM. Bronchiolitis obliterans, bronchiectasis, and other sequelae of adenovirus type 21 infection in young children. J Clin Pathol 1971; 24: 72-82.

15. Cooreman J, Redon S, Levallois M, et al. Respiratory history during infancy and childhood, and respiratory conditions in adulthood. Int J Epidemiol 1990; 19 621-627.

16. Johnston ID, Strachan DP, Anderson HR. Effect of pneumonia and whooping cough in childhood on adult lung function. N Engl J Med 1998; 338: 581-587.

17. Laraya-Cuasay LR, DeForest A, Huff D, et al. Chronic pulmonary complications of early influenza virus infection in children. Am Rev Respir Dis 1977; 116: $617-625$

18. Penn CC, Liu C. Bronchiolitis following infection in adults and children. Clin Chest Med 1993; 14: 645-654.

19. Gratten M, Manning K, Dixon J, et al. Upper airway carriage by Haemophilus influenzae and Streptococcus pneumoniae in Australian Aboriginal children hospitalised with acute lower respiratory infection. Southeast Asian J Trop Med Public Health 1994; 25: 123-131.

20. Torzillo P, Dixon J, Manning K, et al. Etiology of acute lower respiratory tract infection in Central Australian Aboriginal children. Pediatr Infect Dis J 1999; 18 : 714-721.

21. Robinson P. Cystic fibrosis. Thorax 2001; 56: 237-241.

22. Sawyer SM, Robertson CF, Bowes G. Cystic fibrosis: a changing perspective. Aust N Z J Med 1997; 27: 6-11.

23. Ellerman $A$, Bisgaard $H$. Longitudinal study of lung function in a cohort of primary ciliary dyskinesia. Eur Respir J 1997; 10: 2376-2379.

24. Phelan PD, Bowes G. Cystic fibrosis in Melbourne. Thorax 1991; 46: 383-384.

25. Wilson R, Dowling RB, Jackson AD. The biology of bacterial colonization and invasion of the respiratory mucosa. Eur Respir J 1996; 9: 1523-1530.

26. Bush A, Tiddens $H$, Silverman M. Clinical implications of inflammation in young children. Am J Respir Crit Care Med 2000; 162: S11-S14.

27. Stockley RA. Role of bacteria in the pathogenesis and progression of acute and chronic lung infection. Thorax 1998; 53: 58-62.
28. Armstrong DS, Grimwood K, Carzino R, et al. Lower respiratory infection and inflammation in infants with newly diagnosed cystic fibrosis. BMJ 1995; 310 1571-72

29. Shah PL, Mawdsley S, Nash K, et al. Determinants of chronic infection with Staphylococcus aureus in patients with bronchiectasis. Eur Respir J 1999; 14 1340-1344

30. Wilson CB, Jones PW, O'Leary CJ, et al. Effect of sputum bacteriology on the quality of life of patients with bronchiectasis. Eur Respir J 1997; 10: 1754-1760.

31. Wilson R. Bronchiectasis. In: Niederman MS, Sarosi GA, Glassroth J, editors Respiratory infections. 2nd edition. Philadelphia: Lippincott Williams \& Wilkins, 2001; 347-359.

32. Ramsey BW, Pepe MS, Quan JM, et al. Intermittent administration of inhaled tobramycin in patients with cystic fibrosis. N Engl J Med 1999; 340: 23-30.

33. Lin $\mathrm{HC}$, Cheng HF, Wang $\mathrm{CH}$, et al. Inhaled gentamicin reduces airway neutrophi activity and mucus secretion in bronchiectasis. Am J Respir Crit Care Med 1997; 155: 2024-2029.

34. Hill SL, Morrison HM, Burnett D, Stockley RA. Short term response of patients with bronchiectasis to treatment with amoxycillin given in standard or high doses orally or by inhalation. Thorax 1986; 41: 559-565.

35. Burchfiel CM, Marcus EB, Sharp DS, et al. Characteristics associated with rapid decline in forced expiratory volume. Ann Epidemiol 1996; 6: 217-227.

36. Kolbe J, Wells A, Ram FSF. Inhaled steroids for bronchiectasis (Cochrane review). The Cochrane Library 2002; 2. Oxford: Update Software (accessed May 2002).

37. Lewinston NJ. Bronchiectasis in childhood. Pediatr Clin North Am 1984; 31 865-878.

38. De Boeck K. Improving standards of clinical care in cystic fibrosis. Eur Respir J 2001; 16: 585-587.

39. Oldenburg B, McGuffog ID, Turrell G. Socioeconomic determinants of health in Australia: policy responses and intervention options. Med J Aust 2000; 172 489-492.

40. International consultation on environmental tobacco smoke (ETS) and child health, 1999. Available at: http://www5.who.int/tobacco/repository/stp50/ ETS_report.pdf (accessed July 2002).

41. Ezzati M, Kammen D. Indoor air pollution from biomass combustion and acute respiratory infections in Kenya: an exposure-response study. Lancet 2001; 358: 619-624.

42. Cleland JG Van Ginneken JK. Maternal education and child survival in developing countries: the search for pathways of influence. Soc Sci Med 1988; 27: 13571368

43. Kolbe J, Wells AU. Bronchiectasis: a neglected cause of respiratory morbidity and mortality. Respirology 1996; 1: 221-225.

44. Hoque BA, Chakraborty J, Chowdhury JT, et al. Effects of environmental factors on child survival in Bangladesh: a case control study. Public Health 1999; 113: 57-64.

\section{0 common Problems: Geriatrics}

This user-friendly guide is packed with practical solutions to the problems you face daily, and answers the most common health problems seen in the elderly from:

- constipation and skin disorders to memory loss, dizziness, and prostate problems;

- practical guidance on caregiver

issues, polypharmacy, advance

directives, end-of-life medicine;

- illustrated with quick reference charts, useful algorithms and

\section{Common} Problems: Geriatrics Only $\$ 99.95$

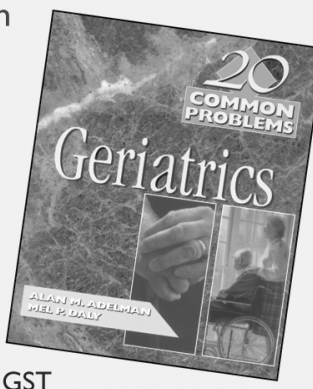

*Plus $\$ 7.65 \mathrm{P} \& \mathrm{H} \cdot$ All prices include GST analyses of diagnostics.

AMA members receive a $10 \%$ discount

For further information contact AMPCo: Ph 0295626666 • Fax 0295626662 Email:sales@ampco.com.au•www.mja.com.au/public/bookroom/
To ORDER, or for further information, contact the Book Sales Coordinator:

AMPCo, Australasian Medical Publishing Co Pty Ltd ABN 20000005854 Locked Bag 3030 Strawberry Hills NSW 2012 • Ph 0295626666 • Fax 029562666 Please send .............. copy(ies) of 20 common problems: Geriatrics @ \$99.95* (*Add \$7.65 Postage and Handling • AMA Members receive a $10 \%$ discount • All prices include GST) To: $\mathrm{Dr} / \mathrm{Mr} / \mathrm{Ms}$ : Address:

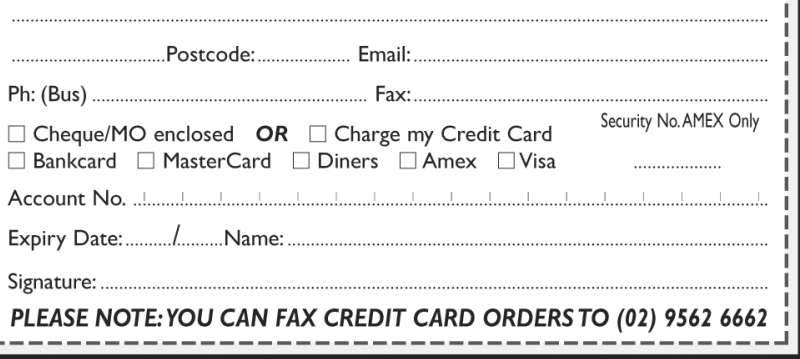

